\section{Subgingival Curettage Versus Surgical Elimination of Periodontal Pockets*}

by

SIGURD P. RAMFJORD

RoBERT R. NISSLE

RichaRD A. SHICK

HUGH COOPER, JR. ANN ARBOR, MICHIGAN

Almost all therapeutic PROCEDURes in periodontics are empirical, based on subjective observations from clinical practice and conceptual speculations. Only during the last few years have interest and tools for clinical trials been developed in periodontics, and such research is still at its inception.

The purpose of the present investigation was to organize and carry out a clinical trial of comparison between results from commonly used methods in the treatment of periodontal pockets. No comparative study of this nature under anything resembling controlled experimental conditions could be found in the literature.

\section{MAterial}

Forty-four patients with advanced periodontal disease involving some or all of their teeth were selected for this study from patients referred to The University of Michigan School of Dentistry. The other criteria besides one or more pockets extending more than $4 \mathrm{~mm}$ apically to the cementum-enamel junction, were that the patients were interested in keeping their teeth and willing to come when called for treatment or examination. Four of these patients have been lost because of death (2), or moving away from the area (2). Of the 40 patients presently participating in the study, 32 have had their periodontal treatment completed long enough to have had at least one reexamination one year following completion of the treatment. Reported here is an analysis of the results of these 32 patients with a total number of 729 treated teeth.

\section{Methods}

At the time this study was initiated, there was no established experimental design available in the dental literature that could be used as an acceptable model for a clinical trial of periodontal therapy. As an initial

\footnotetext{
*This research was supported by U.S.P.H.S. Grant DE 01430 , June 1, 1961 to November 30, 1966.

The University of Michigan School of Dentistry, Department of Periodontics, Ann Arbor, Michigan 48104.
}

approach it was therefore decided to record the periodontal status of the patients in a-number of different ways and by analysis of the results determine the most suitable procedures for such investigations. Besides a medical and dental history with medical examination when it was deemed to be desirable, the following records were obtained for all of the patients: color photographs, periapical and bitewing roentgenograms, cephalograms with the mandible in rest position, casts mounted in an adjustable articulator, electromyographic recordings, bacteriologic samples from the pockets and the mouth, biopsies of pockets, scoring of all teeth with the P.D.I. index, ${ }^{1}$ scoring of gingivitis, ${ }^{1}$ plaque, ${ }^{1,2}$ calculus, ${ }^{1}$ mobility, ${ }^{1}$ attrition, ${ }^{1}$ and open contacts ${ }^{1}$ for all teeth.

Two research associates (R.R.N. and R.A.S.) who both are specialists in periodontics were instructed and calibrated in the use of the various scoring indices. The initial scoring and the rescoring of the same patient during subsequent reexaminations were always done by the same person throughout the study.

All patients initially received comprehensive scaling and root planing, instruction in oral hygiene and occlusal adjustment. During the first 2 years of the study, approximately one half of the patients (Group I) were treated with subgingival curettage after the initial treatment while the other half (Group II) had surgical elimination of the periodontal pockets deeper than $3 \mathrm{~mm}$. This consisted of gingivectomy for pockets that could be eliminated within the attached gingiva and without appreciable bone removal. Repositioning and reverse bevel flaps were used for pockets extending beyond the attached gingiva, pockets deeper than 4 to $5 \mathrm{~mm}$, and for deep palatal pockets. Flap surgery also was used when extensive bone recontouring was needed in order to eliminate the pockets and obtain acceptable gingival contour. An attempt was made to pair patients with similar periodontal status on an equal basis for the two experimental groups.

It became evident after 2 years that assigning one side for curettage and the other side of the same patient's mouth for surgical pocket elimination, on a random basis, would constitute an improvement of the experimental design. This procedure ("split mouth") was used during the last 3 years of the study.

All patients received emergency and maintenance dental care including placement of needed restorations and endodontic therapy. Some patients received needed fixed bridges and a few patients received extensive splinting of loose teeth and replacement of lost teeth by a research associate (H.C.) with extensive experience in restorative dentistry.

The patients were reexamined (utilizing all of the 
procedures included in the initial examination) at the completion of the scheduled treatment. Since the reexamination followed the completion of the planned restorative treatment, several months or a year elapsed before the rescoring of the periodontal status was performed.

The patients were recalled every 3 months for prophylaxis and instruction in home care. An attempt was made to rescore their periodontal status and take new roentgenograms annually after completion of the initial treatment. Unfortunately this schedule was not always kept as rigid as planned during the first part of the study, but was followed for the last 2 to 3 years.

The periodontal data were recorded on scoring sheets, and the scores were transferred to IBM cards for computer analysis. The periapical roentgenograms were scored according to the method of Schei, et al., ${ }^{3}$ and the scores were transferred to the same IBM cards.

\section{Results}

None of the treated teeth were lost during the experimental period. The findings from a total of 729 teeth from the 32 patients were analyzed statistically. A number of variables which might influence the results were included in the analysis: mobility score at the time of initial examination, furcation involvement at the time of the initial examination, initial total pocket depth on the mesial side in $\mathrm{mm}$, and recorded in $\mathrm{mm}$ depth from the C-E junction to the bottom of the pocket on the mesial side. These four variables from the initial examination were compared with the following variables from the latest data available for each patient: gingivitis scores, attrition scores, calculus scores, plaque scores (Ramfjord), ${ }^{1}$ plaque scores (Shick and Ash), ${ }^{2}$ and contact scores.

The differences in scores from the initial examination to the last examination $(\triangle)$ were computed and analyzed for: gain or loss of alveolar bone as scored from the roentgenograms, increase or decrease in total pocket depth in $\mathrm{mm}$ (average of mesial + buccal + distal + lingual measurements). Increase or decrease in modified P.D.I. scores (average of four scores for each tooth), loss or gain in distance from $C$-E junction to the bottom of the mesial pocket measured in $\mathrm{mm}$, and loss or gain in distance from $\mathrm{C}-\mathrm{E}$ junction to the bottom of the buccal pocket measured in $\mathrm{mm}$.

Intercorrelation coefficients were determined and tested for significance for all of the above 14 variables in search for relationships between factors related to periodontal disease and the criteria used to assess the value of treatment.

A number of previously well established relationships were corroborated and found to be statistically significant. Such as: mobility related to pocket depth $(\mathrm{r}=$ $.295, \mathrm{p}<.001$ ), and to depth of the pockets from the C-E junction to the bottom $(\mathrm{r}=.384, \mathrm{p}<.001)$, also highly related were total pocket depth to the distance from the C-E junction to the bottom of the pockets $(\mathrm{r}=.663, \mathrm{p}<.001)$.

At the time of reexamination there was a strong relationship between the gingivitis scores and the plaque scores $(\mathrm{r}=.179, \mathrm{p} .<.001)$, but not so strong a relationship between gingivitis and calculus $(\mathrm{r}=.062)$.

Quantitation of loss or gain of periodontal attachment following treatment was based upon 4 methods: (1) comparison of the measurements of the distance from the C-E junction to the bottom of the mesial pockets measured in $\mathrm{mm}$ before and after treatment (mesial distance), (2) comparison of the measurements from the C-E junction to the bottom of the buccal pockets (buccal distance), (3) differences in modified P.D.I. scores for each tooth before and after treatment, (4) differences between sums of radiographic mesial and distal scores (Schei et al.) ${ }^{3}$ of bone support before and after treatment.

The differences between the mesial distance before and after treatment and the differences in modified P.D.I. scores before and after treatment were strongly related $(\mathrm{r}=.491, \mathrm{p}<.001)$ as were the buccal distance differences with the P.D.I. differences $(r=.501$, $\mathrm{p}<.001$ ) and apparently express very similar values. However, the differences in radiographic scores were not significantly related either to the differences in mesial distance $(\mathrm{r}=.018)$ or to the differences in P.D.I. scores $(r=.003)$, and therefore cannot be considered to express the same values. On the basis of this finding it was decided not to use the roentgenographic scores for the remainder of the analysis of the results.

If the differences in mesial distance were considered alone there was an indication of slight gain of attachment during the treatment period when all teeth were included (Confidence Interval: $-0.111 \pm 0.107$ at 99\% level). The differences in buccal distance were statistically significant and indicate a loss of attachment during the treatment period (CI: $0.342 \pm 0.106$ at 99\% level). However, when the differences in mean P.D.I. scores for all of the teeth before and after the treatment were compared there did not appear to be any significant loss or gain of total attachment of the teeth (CI: $0.013 \pm 0.36$ at $95 \%$ level). In other words, the mesial gains equalized the buccal losses when the total support for the teeth was considered.

When the teeth treated with curettage were placed in a separate group (245 teeth) there was a gain of attach- 
ment from the initial to the last scores if the mean differences in mesial distances (from the C-E junction to the bottom of the mesial pockets) were considered. The gain was statistically significant (CI: $-0.304 \pm 0.188$ at $99 \%$ level). If the changes in buccal distances for the same teeth were considered there was a slight but not statistically significant loss $(\mathrm{CI}:+0.182 \pm 0.205$ at 99\% level).

The differences in mean P.D.I. scores before and after treatment for these curetted teeth indicated gain in attachment when mesial, buccal, distal and lingual measurements were included. The gain was statistically significant $(\mathrm{CI}$ : $-0.66 \pm 0.055$ at $99 \%$ level $)$.

The total result of curettage thus was a slight gain of attachment during the entire treatment.

A similar grouping of all teeth (484) treated by surgical elimination of the pockets was analyzed. When the mean differences of the mesial distance before and after treatment were considered, there was no statistically significant loss or gain (CI: $-0.016 \pm 0.135$ at $99 \%$ level). The mean differences in buccal measurements indicated a loss of attachment for the teeth treated by surgery. The loss was statistically significant (CI: $-0.420 \pm 0.127$ at $99 \%$ level). Also the mean differences in P.D.I. scores showed a loss of attachment which also was statistically significant $(\mathrm{CI}:+0.056 \pm 0.038$ at $99 \%$ level).

This indicates a slight total loss of attachment during the period of treatment for the teeth that had surgical pocket elimination.

\section{Variation of Mean PDI with Time - Total Cases}

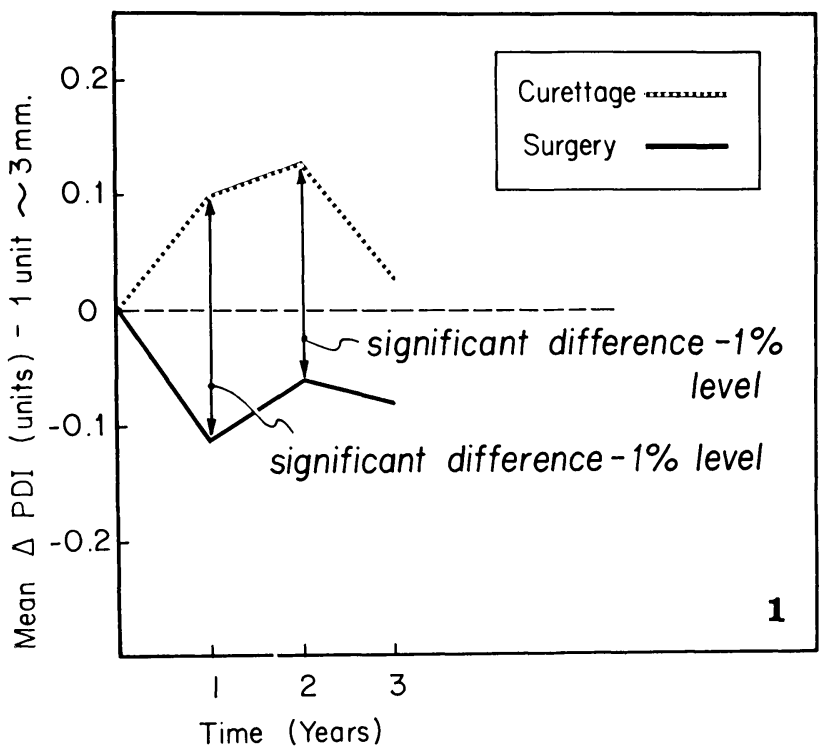

Figure 1.
In order to relate the results of the treatment to time, the mean changes from the initial scores (loss or gain) in P.D.I. scores, mesial distance scores (in $\mathrm{mm}$ ), buccal distance scores (in $\mathrm{mm}$ ) and pocket depth scores (in $\mathrm{mm}$ ) were calculated at the end of yearly intervals. Separate calculations were done for the teeth treated by subgingival curettage and for the teeth treated by surgery. All of the treated teeth were included in this analysis of the mean differences at each time interval.

The variations of the mean P.D.I. scores are indicated in Figure 1. The number of cases were too small for an analysis beyond the 3 year level. At the time of the first reexamination there appears to be a slight loss of attachment following surgery and a slight gain following subgingival curettage. The differences between the gain after curettage and the loss after surgery are statistically significant at the $1 \%$ level for the first two reexaminations. However, the mean differences are only about $0.5 \mathrm{~mm}$. Although the same trend prevails up to the third reexamination, the differences are not statistically significant at that time.

When the mean differences in mesial distance from the C-E junction to the bottom of the pockets are used alone, the curve appears slightly different (see Fig. 2). Again curettage gave the most favorable result, but the difference compared to surgery is statistically significant only for the second reexamination, and the results following surgery show no appreciable gain or loss.

The mean differences in buccal distance from the $\mathrm{C}-\mathrm{E}$ junction to the bottom of the pockets before and after treatment are related to the same time intervals in Figure 3. An average loss of approximately $0.5 \mathrm{~mm}$

\section{Variation of Mean Mesial Distance with Time - Total Cases}

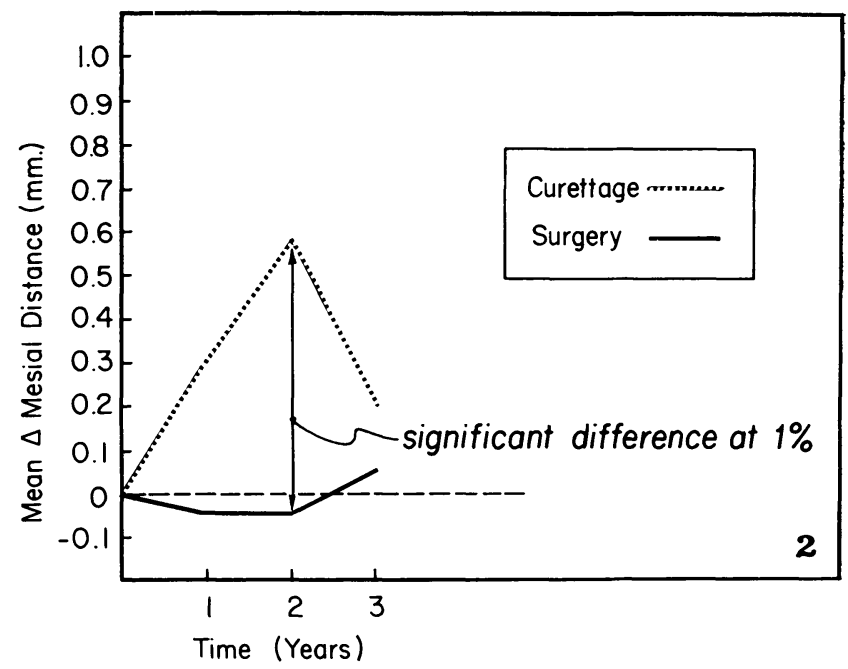

FIGURE 2. 
Variation of Mean Buccal Distance with Time. Total Cases

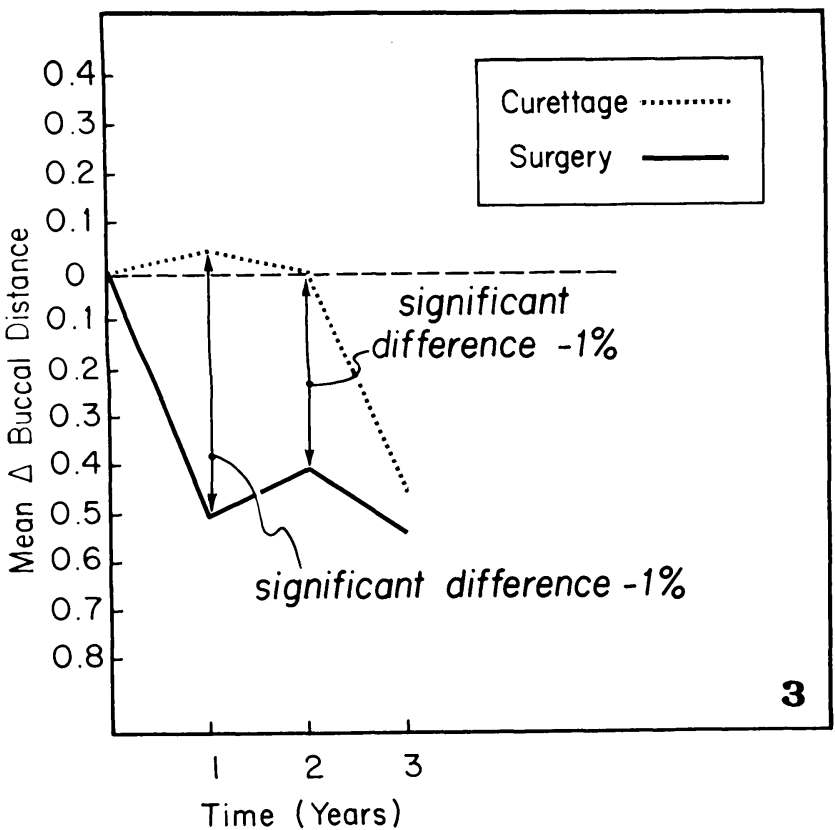

FiguRE 3.

following surgery in contrast to no loss or gain after curettage for the first two reexaminations gave a statistically significant loss of attachment following surgery compared with curettage, but at the third reexamination a loss or attachment for the curetted teeth has almost equalized the results of the two procedures.

If the results are analyzed on the basis of mean reduction of total pocket depth related to the same time intervals (see Fig. 4) the pockets are slightly more reduced in depth at the time of the first reexamination

Reduction of Pocket Depth with Time-Total Cases

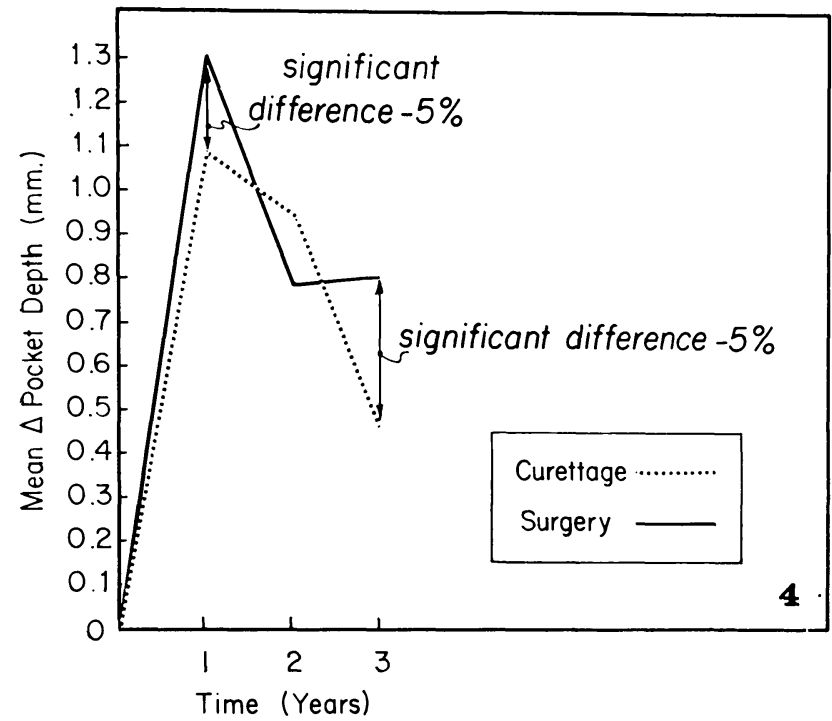

Figure 4.
Variation of Mean PDI with Time-Split Mouth

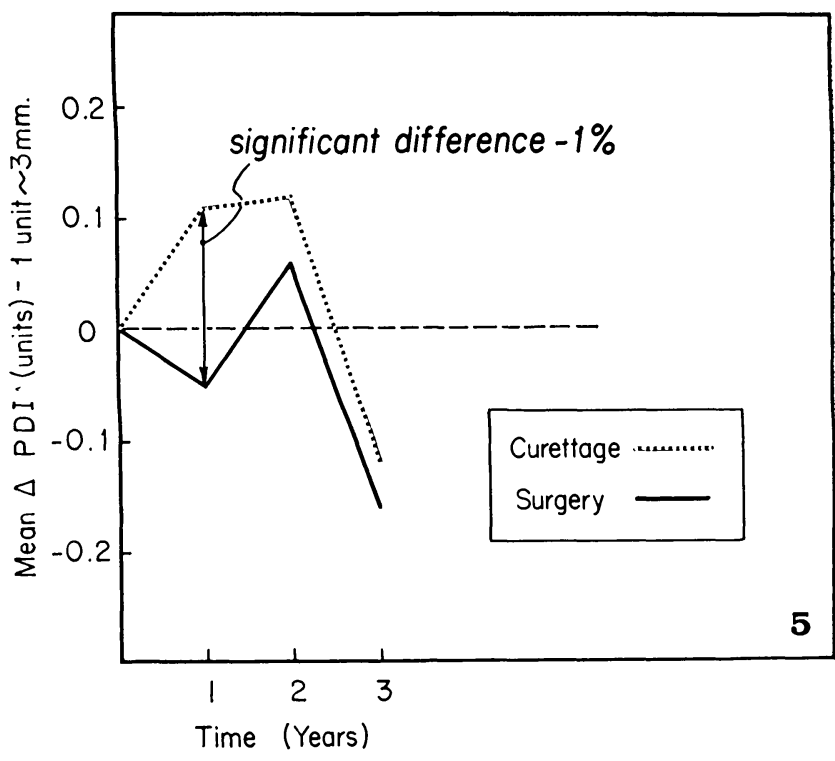

FIGURE 5.

following surgery than following curettage (statistically significant only at the $5 \%$ level and with a mean difference in $0.2 \mathrm{~mm}$ ). At the third reexamination, pocket depth has reoccurred slightly faster after curettage than after surgery. (Again statistically significant only at the $5 \%$ level and mean difference of $0.35 \mathrm{~mm}$ ).

Since all patients treated during the last 3 years have had subgingival curettage on one side of their mouth and surgical pocket elimination on the other side, the results from this "split mouth" approach were analyzed separately. Only 3 patients had more than 2 years of

Variation of Mean Mesial Distance with Time Split Mouth

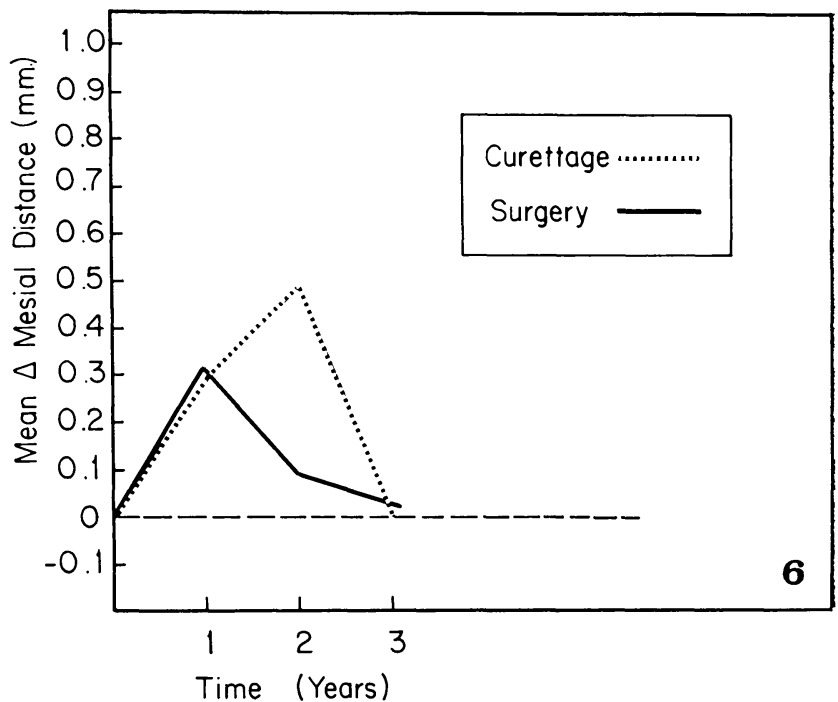

Figure 6. 

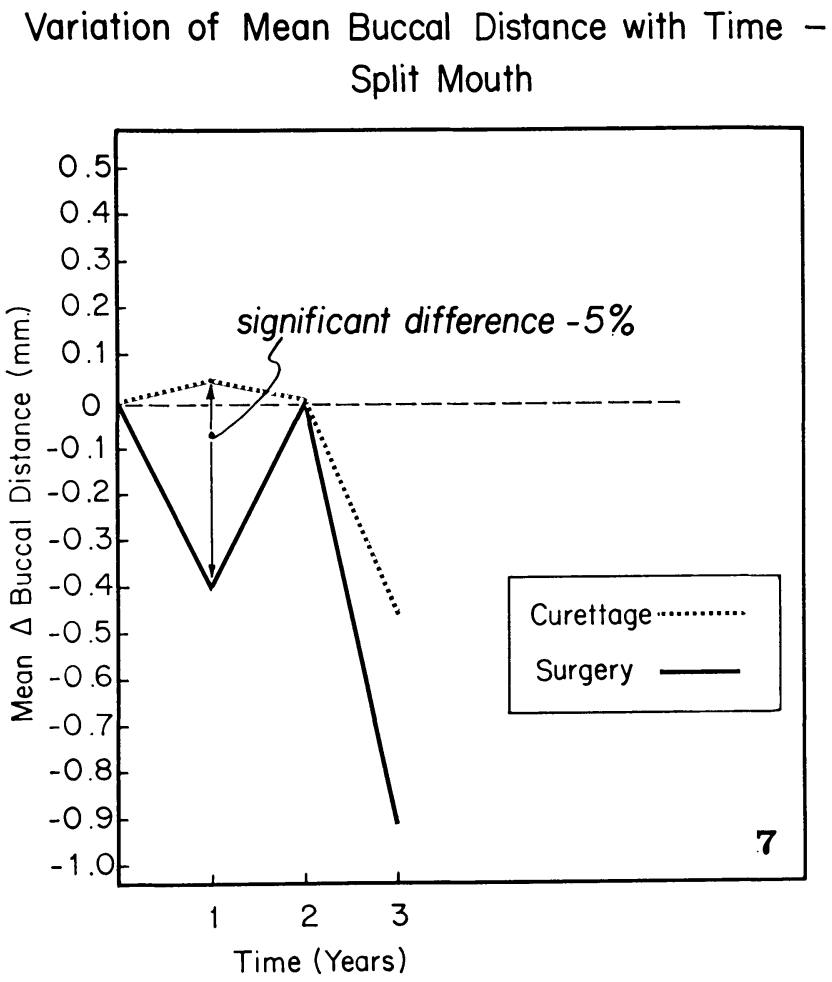

FigURE 7.

follow up after this method was adopted so Figure 5 is really meaningful up to the 2 year level only. It appears that the results of curettage were better than of surgery (statistically significant at the $1 \%$ level) at the time of the first reexamination (a mean difference of approximately $0.5 \mathrm{~mm}$ ). However, at the second and

Reduction of Pocket Depth with Time - Split Mouth

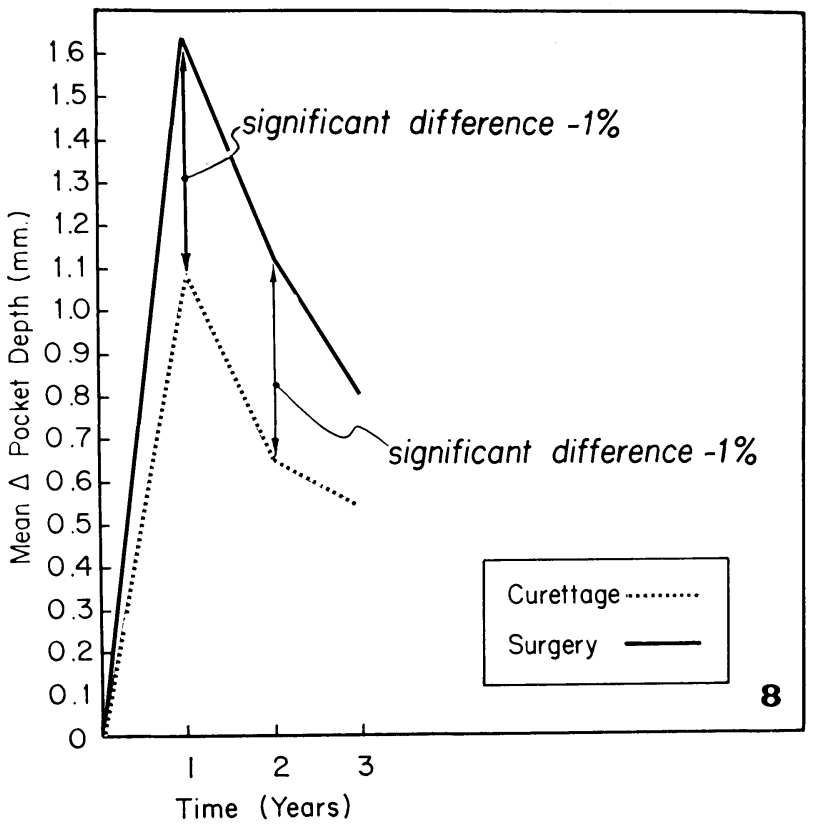

FIgURE 8
Relation of Original Peridontal Loss to Subsequent Variation of Mean Mesial Distance with Time Total Cases

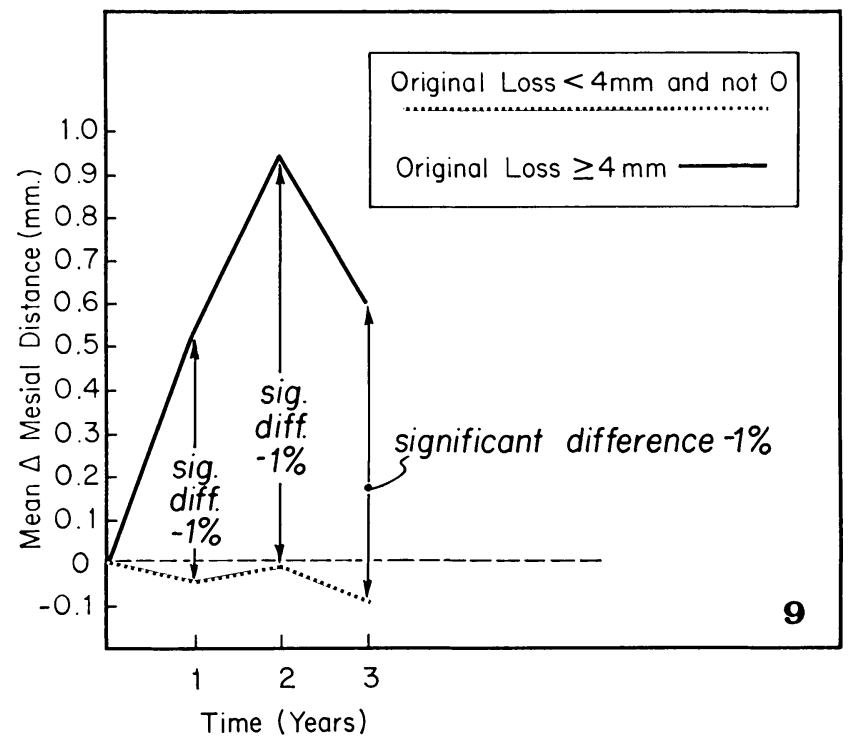

FIGURE 9.

third reexamination the differences were smaller and not statistically significant.

The variations of mean mesial distances from the $\mathrm{C}-\mathrm{E}$ junction to the bottom of the pockets indicated that there was a slight gain of support following both subgingival curettage and surgery (see Fig. 6) but the differences in results from the two methods were slight and not of statistical significance.

Variation of Mean Mesial Distance with Time-Advanced Disease (pockets $>6 \mathrm{~mm}$.)

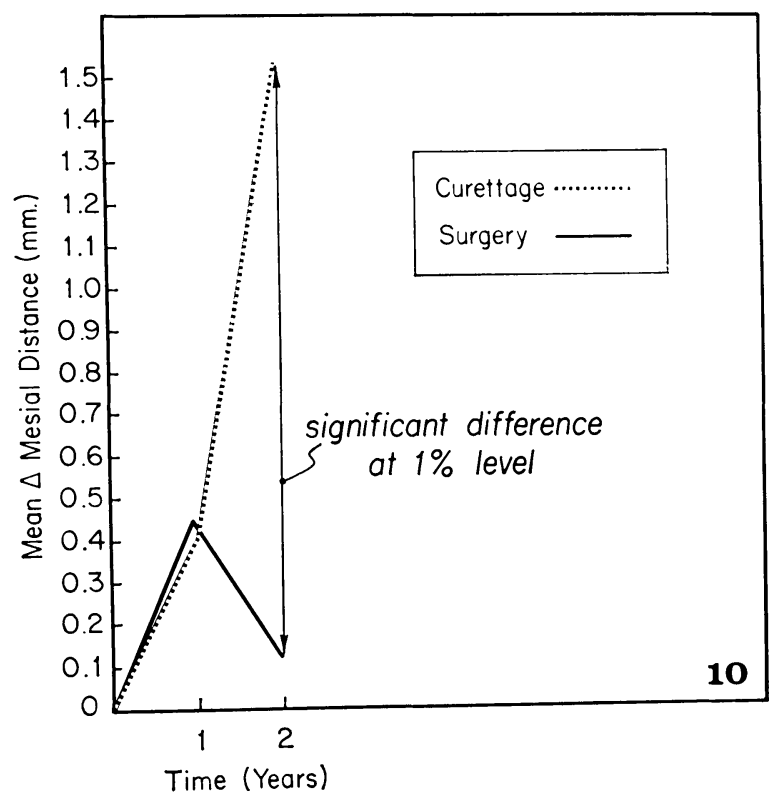

FIGURE 10 
When the variations of the mean buccal distances before and after treatment in the "split mouth" sample were compared (Fig. 7) there appeared to be a slightly greater (statistically significant at the 5\% level) loss of attachment in the surgery group than in the curettage group at the time of the first reexamination (mean about $0.45 \mathrm{~mm}$ ), but this difference did not exist at the second reexamination. No statement of significance can be made for the third reexamination because of the small number of cases.

There was a greater reduction in pocket depth (statistically significant at the $1 \%$ level) after surgery than after curettage both at the first and second reexamination (mean difference approximately $0.5 \mathrm{~mm}$ ) (Fig. 8). However, the trend was toward regaining pocket depth after the first reexamination for both procedures.

A statistically highly significant negative correlation was found between the initial severity of the periodontal disease as expressed in P.D.I. or mesial distance scores and mean changes in periodontal status following treatment (for P.D.I. $\mathrm{r}=-.230, \mathrm{p}<.001$, and for mesial distances $r=.412, p<.001)$. Since this would indicate a more favorable response following treatment of teeth with initial extensive loss of support than in less advanced periodontal disease, all treated teeth with some initial loss of attachment were separated in 2 groups; in one group were included teeth with an initial loss of attachment of 1 to $3 \mathrm{~mm}$. The other group included all teeth with original loss of $>3 \mathrm{~mm}$ (see Fig. 9). While there was practically no loss or gain of support for the teeth with $\leq 3 \mathrm{~mm}$ initial loss of support, the teeth with $>3 \mathrm{~mm}$ loss had gained support at all three reexaminations (statistically significant at the $1 \%$ level, and a mean gain of as much as almost $1 \mathrm{~mm}$ for the second reexamination). If only teeth with a mesial distance between the free gingival margin and the bottom of the pocket of $>6 \mathrm{~mm}$ were analyzed separately (see Fig. 10), there is practically no difference between the results of the two types of treatment at the first reexamination, but at the second reexamination there is a difference in favor of the curettage (statistically significant at the $1 \%$ level and with a mean difference of $1.4 \mathrm{~mm}$ ).

In order to illustrate further the relationship between the initial periodontal status and the results of periodontal treatment (both curettage and surgery), Figure 11 was prepared. The top of the figure indicates the mean loss or gain in $\mathrm{mm}$ of all treated teeth related to their initial attachment level on the mesial side (the column "O" indicates teeth with the epithelial attachment at the C-E junction, the column "1" includes all of the teeth with an initial loss of $1 \mathrm{~mm}$ of attachment as measured from the C-E junction mesially, etc. to 7 $\mathrm{mm}$ of loss). The negative position of columns " $\mathrm{O}$ " to " 2 " indicate a mean loss of attachment for these teeth with initially shallow or no pockets. Columns " 3 " to
Distribution of Mean $\Delta$ Mesial Distance with Original Loss
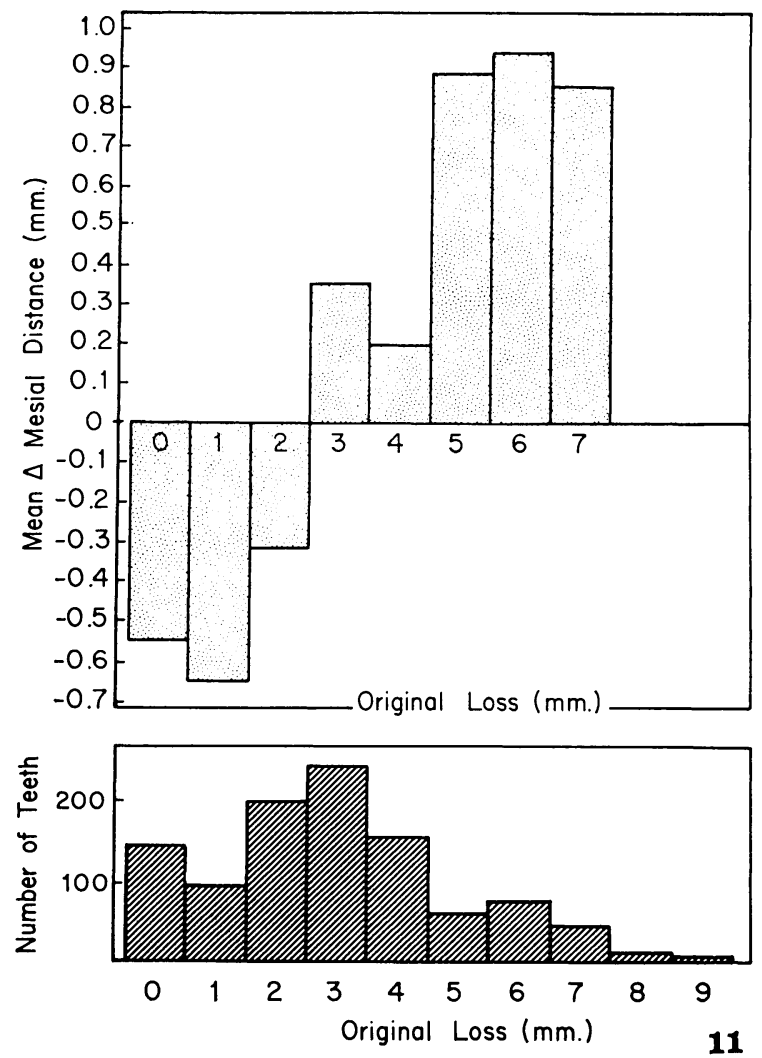

FIGURE 11.

"7" show gain of attachment. This gain becomes markedly significant with an initial loss of support of more than $4 \mathrm{~mm}$.

The number of teeth that fall into each of these columns are indicated at the bottom of Figure 11. The number of teeth with more than $7 \mathrm{~mm}$ of initial loss was too small for analysis.

A separate intercorrelation matrix was generated for the previously listed 14 variables using scores only from the first and second molars. This was done specifically to give better meaning to the correlation between furcation invlovement and subsequent change in pocket depth and loss or gain of attachment. This grouping did not change any of the previously listed relationships, and furcation involvement did not appear to influence the loss or gain of attachment or variations of pocket depth.

The material was also analyzed with regards to gingivitis score after curettage and after surgery. There was no statistically significant difference between the mean gingivitis scores for the curettage and the surgery groups at the reexaminations. The analysis was done both for the total number of teeth and for the "split mouth" ( $1 / 2$ curettage and $1 / 2$ surgery). The follow up mean scores for gingivitis were even closer for the two 
experimental procedures for the "split mouth" (with better control of variables) than for the total sample.

Mean differences in mobility before and after treatment indicated a reduction of mobility when all teeth were considered. The mean reduction (expressed as negative gain) was -0.178 with a confidence interval estimate of $-0.178 \pm 0.055$ significant at the $99 \%$ level of confidence. The change in mobility during the treatment period was not significantly altered by the type of treatment when curettage and surgery were considered separately.

Data from teeth of individuals needing reconstruction and from individuals that received reconstruction were used to generate a correlation matrix with the following variables: splinted teeth, teeth opposing splint, calculus, plaque, change in pocket depth, and change in level of attachment. There was no statistically significant correlation between any of these variables at the $1 \%$ level. There were negative correlations statistically significant at the $5 \%$ level, between splinted teeth and mean loss of attachment (P.D.I) during the treatment period and between teeth opposing splints and loss of attachment (P.D.I.).

This may be interpreted to mean that the periodontal attachment response in splinted teeth and teeth opposing splints were slightly better than for non-splinted teeth. However, a much larger material with much longer time of observation is needed before the clinical significance of such a trend can be assessed.

Several of the recorded data do not lend themselves to scientific analysis and have not been included in this report of results. Information from occlusal analysis of mounted casts, EMG recordings, bacteriologic examination, biopsies, color photographs and attrition scores (altered by occlusal adjustment) defy scientific analysis by methods currently available.

\section{Discussion}

\section{Methods for Collecting Data}

The main purpose of this investigation was to compare in a longitudinal study the results following two basically different approaches for the treatment of periodontal pockets. It has been shown in previous combined clinical and histometric studies ${ }^{4}$ that clinical measurements with a thin probe will accurately (within \pm 0.5 $\mathrm{mm}$ of error) measure the distance between the connective tissue attachment at the bottom of the epithelial attachment and a fixed point on the tooth surface.

It has also been reported that a fairly constant relationship exists between the bottom of the epithelial attachment and the alveolar crest (about $1 \mathrm{~mm}$ ). ${ }^{5}$ The problems associated with assessment of the position of the alveolar crest around teeth from roentgenograms ${ }^{6}$ makes this approach unreliable as a basis for evaluation of relatively small changes in the position of the crest.

It therefore appeared that the most desirable basis for evaluation of results after any treatment, and for evaluation of variance in results from different types of treatment, would be to measure as accurately as possible the level of attachment of the connective tissue at the bottom of the epithelial attachment in relation to the C-E juncion, buccally, mesially, lingually and distally before and after treatment.

Previous calibration tests have shown that for a well trained investigator such measurements can be reproduced accurately with an average deviation per score of $\pm .008 \mathrm{~mm}^{7}$

It is of essential importance to know this error of the method so it can be considered in the final analysis of the results. The recordings of the measurements in millimeters allowed for a much more pinpointed analysis of results, than if the analysis had been made on the basis of the computed P.D.I. indices alone. Although the P.D.I. index gives an accurate estimate of the severity of periodontal disease in an individual or in a population group, it is recommended that for clinical trials involving relatively few subjects, the $\mathrm{mm}$ measurements from the C-E junction are used as a basis for comparison rather than the index, both because smaller units ( 1 instead of $3 \mathrm{~mm}$ ) are used, and the results can be related to the various surfaces of the teeth. The P.D.I. scoring also provides data for study of gingivitis alone without being combined with pocket scores, and thus the relationship of gingivitis to pockets before and after treatment can be studied separately as done in the present study. The role of return of plaque and calculus was also investigated on the basis of the recorded data, and with the 3 monthly scaling, polishing and repeated instructions in home care, the plaque and calculus return did not seem to significantly influence the attachment level of the teeth. For the majority of the patients, the oral hygiene after the initial treatment clinically has to be characterized as good.

\section{Method of Treatment}

In order to achieve reproducible results following clinical procedures it is important that the procedures are described in such detail that they can be reproduced by other investigators.

Since the initial "mouth preparation" was the same for both experimental groups it can be assumed that these procedures were not the basis for the difference in results from the two methods of pocket treatment.

The curettage of periodontal pockets which was carried out under local anesthetics, was a very thorough curettage and included planing of the roots of the teeth 
as well as a vigorous soft tissue curettage with sharp curettes. Only a few teeth were treated at any one appointment and the curetted area was covered by periodontal dressing for one week. At the times of recall (every 3 months), scaling also was carefully extended to the bottom of residual pockets rather than being only a superficial "prophylaxis."

The periodontal surgery was aimed at surgical elimination of periodontal pockets deeper than $3 \mathrm{~mm}$. It involved osteoplasty needed for achievement of desired contour and some osteoectomy, especially for elimination of interproximal craters. The apically repositioned and reverse bevel flaps did not cover any appreciable part of tooth surface and did not cover interproximal areas. A vigorous root planing was included also in the surgical pocket elimination.

The gingivectomies for less extensive pockets were extended to the bottom of the clinically measurable pocket, which as shown in a previous paper will involve removal of the epithelial attachment. ${ }^{4}$

Therefore, on the basis of the detailed, vigorous curettage and type of periodontal surgery, it has to be assumed that the crevicular epithelial lining and the epithelial attachment were removed both for the curetted and the surgically eliminated pockets.

\section{SIGNIFICANCE}

By far the most significant finding in this investigation is the gain of attachment that occurred following curettage and periodic scaling in deep periodontal pockets. The clinical significance of this promising observation can be assessed only by longer periods of follow up and treatment of more patients. However, it should be noted that Lovdal and co-workers ${ }^{8}$ also found very good results following scaling and instruction in home care in the only other controlled longitudinal study that could be found in the literature.

The statistically significant total gain of attachment following subgingival curettage compared with a slight loss following surgical pocket elimination obviously will call for revision of current prevailing periodontal practice, if these results are confirmed by more extensive studies.

On the basis of the results of this study it appears that teeth with deep periodontal pockets (greater than $6 \mathrm{~mm}$ ) are more successfully treated with subgingival curettage than with attempts on surgical elimination of the pockets.

As could be expected, surgical treatment reduced pocket depth more than curettage but the trend was toward recurrence of pocket depth both following surgery and curettage, although to a lesser degree after surgery. Clinically, the two posterior parts of the mouth 2 to 3 years after treatment appear identical, while in the incisor regions more root surface will remain exposed after surgery than after curettage.

The lowering of the buccal attachment level as measured from the C-E junction shortly after surgery may be explained on the basis of removal of buccal and lingual supporting tissues, including bone, in order to eliminate interproximal pockets and to gain acceptable contour. There appeared to be a trend toward gradual regeneration of such supporting tissues that had been removed surgically. This observation casts doubt upon the rationale of removing buccal or lingual bone and soft tissue support to the level of interproximal craters as commonly has been done during surgical pocket elimination.

In these relatively short term observations, furcation involvement did not seem to affect the loss or gain of periodontal support in molar teeth.

From this very limited material it does not appear to be very important for maintenance of the level of the periodontal attachment whether teeth with extensive loss of periodontal support are splinted together or not; but it should be emphasized that this study did not include sufficient material to make significant conclusions regarding the value of splinting of teeth.

No limits were established for severity or degree of periodontal disease beyond which treatment can not evoke a favorable response with clinical evidence of some gain of attachment.

This study is being continued for another 3 years under a new U.S.P.H.S. grant.

\section{CONCLUSIONS}

1. Gain of periodontal attachment occurred following curettage of deep periodontal pockets. The gain was statistically significant.

2. Subgingival curettage was followed by more favorable results than surgical elimination of periodontal pockets. The difference was statistically significant.

3. Slight loss of attachment followed surgical elimination of periodontal pockets.

4. A satisfactory method has been developed for longitudinal study of the results of various forms of periodontal treatment.

\section{ACKNOWLEDGMENT}

The computations and the statistical analysis of all data included in this paper were directed by Dr. James W. Knowles, The University of Michigan School of Dentistry, Ann Arbor, Michigan. 


\section{REFERENCES}

1. Ramfjord, S.: Indices for Prevalence and Incidence of Periodontal Disease. J. Periodont., 30:51-59, Jan., 1959.

2. Shick, R. A. and Ash, M. M., Jr.: Evaluation of the Vertical Method of Toothbrushing. J. Periodont., 32:346353, Oct., 1961.

3. Schei, O., Waerhaug, J., Lovdal, A. and Arno, A.: Alveolar Bone Loss as Related to Oral Hygiene and Age. J. Periodont., 30:7-16, Jan., 1959.

4. Ramfjord, S. P. and Costich, E. R.: Healing after Simple Gingivectomy. J. Periodont., 34:401-415, Sept., 1963.

5. Stanley, H. R., Jr.: The Value of Step-Serial Sectioning in the Morphologic Study of Human Periodontal Disease. J. Periodont., 28:248-252, July, 1957.

6. Theilade, J.: An Evaluation of the Reliability of Radiographs in the Measurement of Bone Loss in Periodontal Disease. J. Periodont., 31:143-153, April, 1960.

7. Smith, W. A. and Ash, M. M., Jr.: A Clinical Evaluation of an Electric Toothbrush. J. Periodont., 34:127-136, March-April, 1964.

8. Lovdal, A., Arno, A., Schei, O. and Waerhaug, J.: Combined Effect of Subgingival Scaling and Controlled Oral Hygiene on the Incidence of Gingivitis. Acta Odont. Scand., 19:537-555, Dec., 1961.

\section{Abstracts}

\section{Bone Induction by DeCalcified Dentine Implanted into Oral, Osseous and Muscle Tissues}

Yeomans, J. D. and Urist, M. R.

Arch. Oral Biol. 12:999-1008, 1967

The object of this experiment was to determine the bone induction effect of implanting decalcified cortical bone, decalcified dentine, tendon and muscle into three receptor sites of young adult New Zealand rabbits: a) a pouch in the rectus abdominus muscle, b) a drill-hole bone defect in the mandible, and c) an empty tooth socket. With the implants of dentine and bone, a consistently reproducible induction system was set up for osteogenesis which was not found with the tendon and muscle implants. The new bone developed from the interaction of hypertrophied mesenchymal cells with extracellular substances in the matrix of dentine and bone. A film of cement substance generally separated the old and new bone. University of California, Los Angeles, California.

The Adsorption of Salivary Proteins By Hydroxyapatite aNd ENAMEL

Hay, D. I.

Arch. Oral Biol. 12:937-946, 1967

Studies of the adsorption of salivary proteins from whole saliva on to hydroxyapatite and enamel powder revealed highly selective adsorption of a few of the salivary proteins. The adsorption behavior of the proteins was followed electrophoretically. Protein was also recovered and studied from freshly extracted teeth to compare with the in vitro results. The carbohydrate components of the adsorbed proteins were analyzed by hydrolysis with $\mathrm{N} \mathrm{HCL}$ for two hours at $100^{\circ} \mathrm{C}$. To ascertain the effect of mucin as a component of the pellicle, the apatite: saliva ratio was analyzed with $10 \mathrm{ml}$. of saliva to 50,200 and $350 \mathrm{mg}$. of apatite. The supernatants were analyzed for sialic acid using Warren's method. The results showed the adsorbed proteins to contain glucose, galactose, mannose, fucose, glucosamine and galactosamine. Proteins from extracted teeth and the proteins adsorbed on to hydroxyapatite and powdered enamel have the same electrophoretic mobility. It seems probable that these proteins are identical. Sialoprotein was not found to be selectively adsorbed implying that mucin, if involved in pellicle formation, has been changed perhaps by bacterial activity or may not be involved in early pellicle formation. Forsyth Dental Center, Boston, Massachusetts.
The EfFect of Sex Hormones on Inflammation. II. Progestogen, Oestrogen, and Chorionic Gonadotropin

Lindhe, J. and Sonesson, B. J. Perio. Res. 2:7-12, 1967

A comparative study of the effect of the sex hormones progestogen, oestrogen, and chorionic gonadotropin on acute connective tissue inflammation induced by croton oil was conducted using a modified granuloma pouch technique on 88 female Wistar rats. If any of the sex hormones used was deposited immediately prior to the croton oil, a noticeably less extensive inflammation occurred. It was concluded that the sex hormones act as antiphlogistic substances on the acute inflammatory process induced by the croton oil. This information cannot be applied clinically, but can serve as a theoretical basis for further study. Departments of Anatomy and Periodontology, School of Dentistry, University of Lund, Lund, Sweden.

\section{Enzyme Treatment of Traumatic Swelling in} Oral and Maxillofacial Surgery

Wigand, F. and Messer, E. Clinical Medicine 74:29-32, July 1967

The anti-inflammatory effectiveness of crystalline chymostrypsin, a proteolytic enzyme, in reducing traumatic edema following surgery was tested clinically on 56 patients ( 18 females and 38 males) ranging from nine to 59 years of age: 22 received ex. traction, cystectomy, apicoectomy or miscellaneous other oral surgical procedures, and had accidental maxillofacial trauma usually involving fractures. Each patient was given one or two tablets $(20 \mathrm{mg}$. per tablet) of crystalline chymotrypsin, four times a day, started immediately after surgery or upon arrival and continued to a usual total dosage of 24 tablets. A comparison of actual degree of swelling with the normally expected degree of swelling was made and a first day score of two points was assigned as the normally expected degree of swelling in all routine surgical cases, based on previous experience. Against these scores were recorded the actual scores observed on the first five days, graded as $0=$ none, through $4=$ excessive. By comparing the first day actual score against the first day "guess" score, the traumatic swelling after 24 hours was about onethird of that normally expected. These patients recovered more rapidly and the need for analgesic drugs after the first 24 hours appeared less than usually required in these cases. U. S. Naval Hospital, Great Lakes, Illinois. 